\title{
Preface: Plasma Sources for Biological and Biomaterial Applications
}

Plasmas have been used as sources for electrons, ions, excited atoms and molecules, free radicals, transient electric fields, and light of various wavelengths. Designing and optimizing a plasma source for particular purposes is often an integral and crucial part of the technological development of plasma processing. In biological applications, such as in vitro/in vivo experiments or clinical applications of plasma medicine, reactive oxygen and nitrogen species (RONS) generated by a plasma directly or indirectly (via chemically reactive liquid media modified by the plasma) interact with biological surfaces such as cell membranes or surfaces of tissues and organs. In biomaterial applications, energetic ions, free radicals, and possibly ultraviolet light that are generated in plasma modify the surfaces of biomaterials by functionalization or deposition of coatings. In either case, the outcome may sensitively depend on how efficiently the plasma source can provide the reactive species necessary for the process of our interest.

In this special issue, we present nine excellent articles discussing various plasma sources that can be used for medical/agricultural applications or biomaterial processing. Most articles in this special issue discuss plasma sources of novel design or innovative use of existing plasma systems. While, for some medical studies, detailed and longterm research using standardized medical devices with particular plasma source designs is imperative, the emergence of new applications will continue to drive innovation in plasma source technologies. We hope that this special issue keeps the reader up to date on the latest developments in this exciting field.

Guest Editors:

Farzaneh Arefi-Khonsari

Sorbonne Université

Paris, France

Satoshi Hamaguchi

Osaka University

Osaka, Japan

Eric Robert

Université d'Orléans

Orléans, France 
